\title{
Removal of Base-Line Wander and Power-Line Interference from the ECG by an Efficient FIR Filter with a Reduced Number of Taps
}

\author{
J. A. VAN ALSTÉ, MEMBER, IEEe, AND T. S. SCHILDER
}

\begin{abstract}
Linear phase filtering is proposed for the removal of baseline wander and power-line frequency components in electrocardiograms. In order to reduce the large number of computations involved in the digital filtering that are necessary, the desired filter spectrum was defined periodically. Making use of the property that the spectrum period is $50 \mathrm{~Hz}$, the spectrum can be realized with a considerably reduced number of impulse response coefficients. This, in combination with the necessary impulse response symmetry, leads to a reduction in the number of multiplications per output sample by a factor of 10 . A suitable impulse response is designed with a pass-band ripple of less than $0.5 \mathrm{~dB}$ and a high stop-band attenuation. The applicability is demonstrated by applying the filtering to exercise electrocardiograms.
\end{abstract}

\section{INTRODUCTION}

$\mathrm{T}$ HE electrocardiogram (ECG) obtained during graded exercise testing of a subject on a bicycle ergometer or treadmill is often contaminated by disturbances such as power-line frequency components and base-line wander. The removal of these disturbances is one of the first steps in the processing of the ECG; not only before further automatic processing, but also as a first step in visual diagnosis. Its purpose is to make the processing easier or even possible and to enable reliable $S T$ segment measurements. Meijer [1] describes a fast and elegant technique for the computation of a third-order polynomial through consecutive $P R$ segments. This cubic spline technique gives a rather good estimation of the base-line wander in normal ECG recordings. The method assumes that the $P R$ segments are well defined, recognizable, and that their position is known. When not, splining methods fail. A useful method for removing power-line and base-line disturbances in real time is the application of digital linear phase filtering [2], [3]. A major drawback of this method is the long computation time caused by the large number of multiplications involved in the filtering in the time domain. The computation time problem is mainly caused by the linear phase narrow-band filtering that requires a long impulse response and a corresponding number of filter coefficients. When using digital nonrecursive finite impulse response filters (NRFIR), a symmetrical impulse response

Manuscript received January 7, 1985; revised April 6, 1985.

The authors are with the Twente University of Technology, Department of Electrical Engineering, Bioinformation Group, Enschede, The Netherlands. can be realized, having the property that the phase is a linear function of frequency which corresponds with an exact delay time [4].

An alternative is filtering in the frequency domain which is also time consuming and introduces long computational delays and transients, and is not very suitable for real-time processing.

Our approach is to reduce the computation time by reducing the number of coefficients of the impulse response. Therefore, we defined the desired frequency spectrum periodic with small stop-band notches, at $0 \mathrm{~Hz}$ to remove base-line wander, as well as at $50 \mathrm{~Hz}$ and at its higher harmonics to remove power-line frequency disturbances. As a result of the spectrum periodicity, a lower sampling rate can be used for the impulse response than for the ECG in- and output signals. In this way, the number of multiplications per output sample is greatly reduced compared to the standard direct-form implementations.

\section{BAND-PASS Filtering OF THE ECG}

An important problem in the high-pass filtering of ECG's, in order to remove base-line wander, is how much to filter. Or stated otherwise, what is the significance of the low-frequency components of the ECG signal? When a bradycardia of 48 beats per minute is considered as the lowest heart rate to be processed, and the ECG is considered to be perfectly periodic, then the lowest frequency component of the ECG spectrum is $0.80 \mathrm{~Hz}$. The ECG spectrum is continuous, however, due to the aperiodicity of the ECG.

Convenient cutoff frequencies for high-pass filtering were obtained in a previous study [2] where exercise ECG's were filtered with an almost rectangular digital high-pass filter and the removed signal part was studied for the presence of components correlated with the heartbeat. In this way, problems in estimating the low-frequency properties of the ECG were avoided. The conclusion drawn from that study, and from the ECG signals processed for this study, was that in spite of the aperiodicity of the ECG, the low cutoff frequency may be as high as the heartbeat frequency without disturbing the waveform of nonectopic beats.

In case of a filter with a fixed low cutoff frequency, this frequency can be chosen as $0.8 \mathrm{~Hz}$ (corresponding to 48 


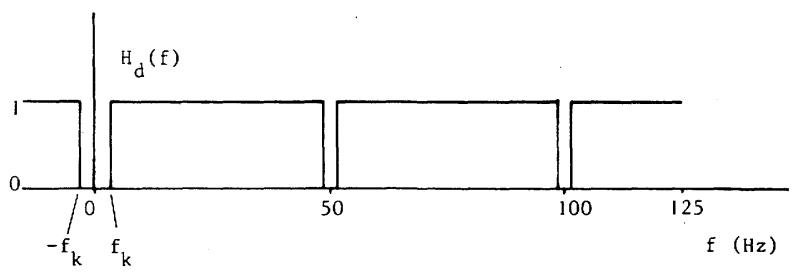

Fig. 1. Desired frequency characteristic of the filter for removal of baseline wander, $50 \mathrm{~Hz}$ power-line frequency components and their higher harmonics.

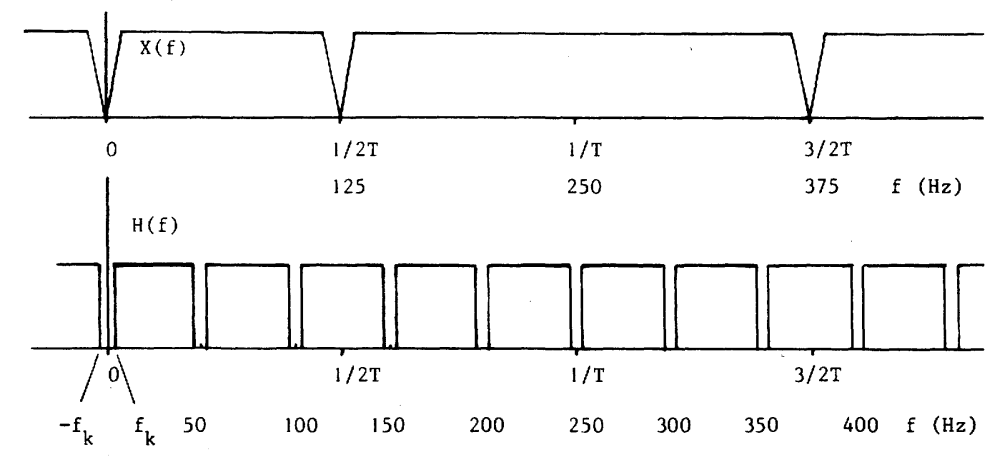

Fig. 2. Periodic spectra of input signal $x$ and impulse response $h$, resulting from sampling them at a rate of $250 \mathrm{~Hz}$. The sampling interval $T$ is thus $4 \mathrm{~ms}$.

bpm, bradycardia) or lower. This is in contrast to the American Heart Association recommendations for ECG recordings [5] which state that frequency components above $0.05 \mathrm{~Hz}$ should not be removed. Evidently, these recommendations have been based on filter methods that introduce phase distortions above the heartbeat frequency. This is the case when using analog filters or recursive digital filters with base-line suppressing properties. Conforming to the American Heart Association recommendations, the cutoff frequency is specified at the $-0.5 \mathrm{~dB}$ point, and the pass-band ripple is not allowed to exceed $\pm 0.5 \mathrm{~dB}$.

The removal of base-line wander is not the only desired property of the filter. Another one is the removal of $50 \mathrm{~Hz}$ power-line frequency noise and its higher harmonics.

The sample frequency of the input ECG signals is taken as $250 \mathrm{~Hz}$. Signal components of the ECG signals with frequencies higher than $125 \mathrm{~Hz}$ are removed by an analog presampling low-pass filter. Now the desired frequency characteristic $H_{d}$ of the filter is defined. (See Fig. 1.) Definition of the spectrum in the area with $f>125 \mathrm{~Hz}$ (and $f<-125 \mathrm{~Hz}$ ) is not necessary because these frequency components are not present in the input signal and are rejected by the output reconstruction filter after the digital to analog conversion.

The sample frequency was chosen as a multiple of the power-line frequency. In case of a power-line frequency of $60 \mathrm{~Hz}$, the sample frequency would be $300 \mathrm{~Hz}$ and the reasoning is valid again.

The cutoff frequency $f_{k}$ specifies the width of the stopband for the base-line wander removal. A suitable value is
$0.7 \mathrm{~Hz}$. The width of the stop-bands at 50 and $100 \mathrm{~Hz}$ will be discussed later.

\section{Filter Method}

The applied filter method is the discrete convolution of the input signal with the impulse response coefficients of an NRFIR filter. The convolution is described by (1).

$$
y(n T)=\sum_{i=0}^{N-1} x((n-i) T) \cdot h(i T)
$$

where

$T=$ sampling interval,

$y(n T)=$ output signal samples,

$x(n T)=$ input signal samples,

$h(i T)=$ filter impulse response coefficients,

$N=$ number of filter coefficients.

By convolving the input signal $x$ with the impulse response $h$, the corresponding spectra can be multiplied. Because $x$ and $h$ are discrete time signals with time interval $T$ between two successive elements, both spectra are periodic with period $1 / T \mathrm{~Hz}$ as shown in Fig. 2. The spectrum $H(f)$ is obtained by periodic repetition of $H_{d}(f)$ from Fig. 1.

\section{Reduction of Computations}

It can be seen from (1) that every output signal sample $y(n T)$ requires $N$ multiplications and summations. This number can be reduced by reducing the number of impulse-response coefficients and by using the symmetry of the impulse response. 


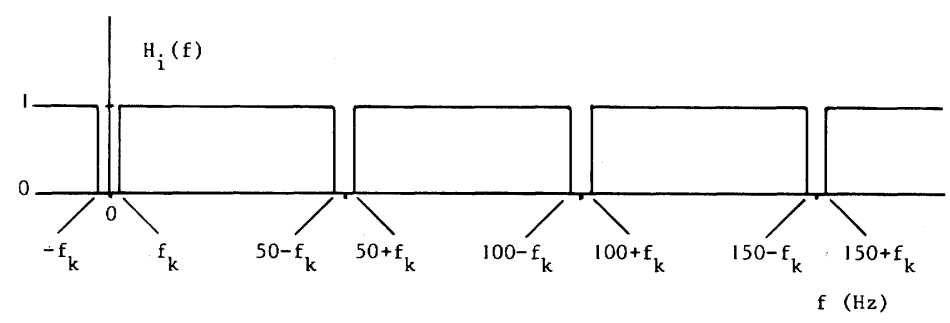

Fig. 3. Desired filter characteristic from Fig. 1 now with a $50 \mathrm{~Hz}$ period.

\section{The Reduction of the Number of Filter Coefficients}

Assume the number of filter coefficients is reduced from $N$ to $M$. When the impulse response length $(N-1) T$ is chosen to be constant, then a reduction of the number of coefficients causes longer time intervals between successive coefficients. [See (2).]

$$
\begin{gathered}
(N-1) T=(M-1) k T \\
\quad \text { (equal impulse-response length) } \\
M=\frac{N-1}{k}+1
\end{gathered}
$$

where

$$
\begin{aligned}
M= & \text { reduced number of filter coefficients } \\
k= & 1,2,3,4, \cdots \\
k T= & \text { increased time interval between two successive } \\
& \text { impulse-response coefficients in the case of their } \\
& \text { number being reduced. }
\end{aligned}
$$

The factor $k$ must be a natural number to make the convolution effective. If the time interval is $k T$, the period of the filter frequency response decreases from $1 / T$ to $1 / k T$.

A closer examination of $H(f)$ in Fig. 2 reveals that it becomes periodic with a period of $50 \mathrm{~Hz}$ when the width of the stopbands at $50 \mathrm{~Hz}$ and its higher harmonics are chosen to be $2 f_{k}$. (See Fig. 3.)

In order to obtain a periodic frequency response with a period of $50 \mathrm{~Hz}$, the interval between two successive filter coefficients must be $1 / 50 \mathrm{~s}$. When the input signal is sampled at a rate of $250 \mathrm{~Hz}$, being exactly 5 times the spectrum period, then the number of filter coefficients can be reduced by a factor 5 , using the periodicity property.

In the case of a $60 \mathrm{~Hz}$ power-line frequency, a signal sampling rate of $300 \mathrm{~Hz}$ would be a suitable choice.

Because the time intervals between impulse response coefficients are now a multiple of that of the input signal samples, the convolution sum can be written in a different form as follows:

$$
y(n T)=\sum_{i=0}^{M-1} x((n-k i) T) \cdot h(i k T)
$$

where

$$
\begin{aligned}
& T=\text { sampling interval of both the input and the } \\
& \text { output signal } \\
& k=5
\end{aligned}
$$

$$
\begin{aligned}
k T= & \text { time interval between successive impulse re- } \\
& \text { sponse coefficients } \\
x(n T)= & \text { input signal } \\
y(n T)= & \text { output signal } \\
h(i k T)= & \text { filter impulse-response coefficients } \\
M= & \text { number of filter coefficients. }
\end{aligned}
$$

\section{The Symmetry of the Impulse Response}

In the next section, it will be shown that the impulse response has to be symmetrical around a certain point $M$ so

$$
h(i k T)=h(M-1-i k T) .
$$

This property can be used for a further reduction of the number of computations. The convolution can be described as follows:

$$
\begin{aligned}
y(n T)= & \sum_{i=0}^{(M-3) / 2} h(i k T) \cdot\{x((n-k i) T) \\
& +x((n-k(M-1)+k i) T)\} \\
& +h\left(\left(\frac{M-1}{2}\right) k T\right) \cdot x\left(n-k\left(\left(\frac{M-1}{2}\right) T\right) .\right.
\end{aligned}
$$

Now instead of $M$, only $(M-3) / 2+2$ multiplications have to be carried out.

When an impulse-response length of $1 \mathrm{~s}$ is taken, conventional FIR filtering requires 251 multiplications and additions every $4 \mathrm{~ms}$. By using a filter frequency characteristic with a $50 \mathrm{~Hz}$ period this number can be reduced to 51 using (2). Using the impulse-response symmetry the number of multiplications becomes 26 . Thus, the number of multiplications is reduced by a factor of about 10 , and the number of additions by a factor of about 5 .

\section{Determination of the Filter IMPULSE-RESPONSE COEFFICIENTS}

A formulation of the spectrum depicted in Fig. 3 is

$$
\begin{aligned}
H(f) & =0 \quad \text { for } \quad|f-n \cdot 50|<f_{k} ; \\
n & =\cdots,-2,-1,0,1,2, \cdots, \\
H(f) & =1 \quad \text { anywhere else. }
\end{aligned}
$$

The periodicity in the spectrum allows development into 


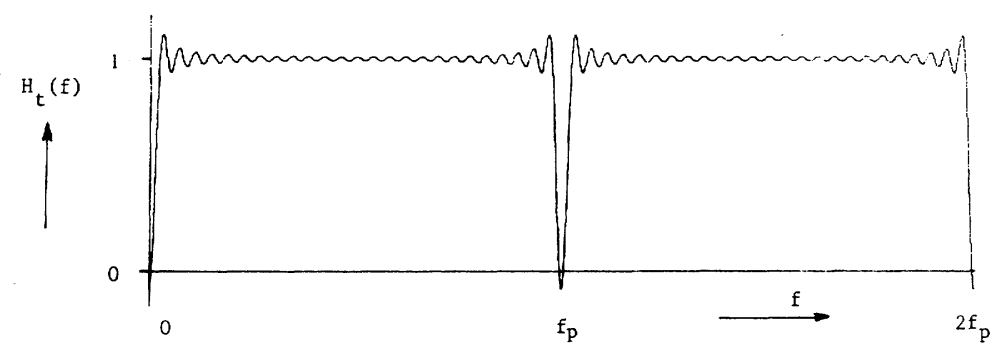

Fig. 4. The distorted frequency spectrum as derived from a truncated impulse response. Note that the scales are linear.

a Fourier series. The Fourier series can be written as

$$
\begin{aligned}
H(f)= & \frac{a_{0}}{2}+\sum_{n=1}^{\infty}\left(a_{n} \cos \left(2 \pi f n T_{p}\right)\right. \\
& \left.+b_{n} \sin \left(2 \pi f n T_{p}\right)\right)
\end{aligned}
$$

where

$$
a_{n}=\frac{2}{f_{p}} \int_{-f_{p / 2}}^{f_{p / 2}} H(f) \cos \left(2 \pi f n T_{p}\right) d f
$$

and

$$
b_{n}=\frac{2}{f_{p}} \int_{-f_{p / 2}}^{f_{p / 2}} H(f) \sin \left(2 \pi f n T_{p}\right) d f
$$

in which $f_{p}=1 / T_{p}$ is the period of the spectrum. Because of the symmetry of $H(f)$ with regard to $f=0$, all coefficients $b_{n}$ are zero. Substitution of (6) in (8) gives

$$
\begin{aligned}
& \frac{a_{0}}{2}=1-\frac{2 f_{k}}{f_{p}} \\
& a_{n}=-2 \frac{\sin \left(2 \pi n f_{k} T_{p}\right)}{\pi n} .
\end{aligned}
$$

Inverse Fourier transformation of the Fourier series describing the continuous spectrum (6) with coefficients given in (10) results in a discrete infinite noncausal impulse response $h_{c}$

$$
\begin{aligned}
h_{c}(0) & =\frac{a_{0}}{2}=1-2 \frac{f_{k}}{f_{p}} \\
h_{c}\left(n T_{p}\right) & =h_{c}\left(-n T_{p}\right)=\frac{a_{n}}{2}=\frac{\sin \left(2 \pi n f_{k} T_{p}\right)}{\pi n} \\
n & =\cdots,-3,-2,-1,0,1,2,3, \cdots, \\
h_{c} & =0 \quad \text { anywhere else. }
\end{aligned}
$$

This impulse response is symmetric as a result of the reality of the spectrum defined in (6).

In order to obtain from (11) an impulse response which can be implemented as an NRFIR filter, the impulse response must be finite and causal.

After truncation, the impulse response becomes finite with length $M T_{p}$, which is achieved by limitation of $n$ in (11) as follows:

$$
|n| \leq(M-1) / 2
$$

where $M$ is odd.
The truncation is performed symmetrically with respect to $n=0$ in order to maintain a symmetrical impulse response with the corresponding real spectrum $H_{t}(f)$ as shown in Fig. 4. The truncation of the discrete impulse response causes distortion of the desired frequency spectrum of the filter. The filter becomes causal by shifting the impulse response over a time interval with length $(M-1) T_{p} / 2$. This operation introduces an exact delay between output and input of the filter which is also $(M-$ 1) $T_{p} / 2$. After the shift operation, the spectrum $H_{t}(f)$ of the truncated impulse response becomes $H_{t p}(f)$

$$
H_{t p}(f)=e^{-j 2 \pi f(M-1) T_{p} / 2} H_{t}(f) .
$$

The resulting impulse response is presented in (14) and the corresponding frequency spectrum $H_{t p}(f)$ is according to $H_{t}(f)$ presented in Fig. 4 .

$$
\begin{gathered}
h\left(n T_{p}\right)=\frac{\sin \left(2 \pi\left(n-\frac{M-1}{2}\right) f_{k} T_{p}\right)}{\pi\left(n-\frac{M-1}{2}\right)} \\
0 \leq n \leq M-1 \\
n \neq \frac{M-1}{2} \\
M=\text { odd } \\
h\left(\frac{M-1}{2} T_{p}\right)=1-\frac{2 f_{k}}{f_{p}} \\
h=0 \quad \text { anywhere else. }
\end{gathered}
$$

\section{Design Adjustments}

The obtained filter needs improvement in two aspects;

1) the pass-band ripple must be reduced, and

2) the attenuation in the stop-bands must be increased.

\section{Improvement of Pass-Band Ripple}

To improve the pass-band ripple, the impulse response (14) is multiplied by a Kaiser window [4].

$$
h_{w}\left(n T_{p}\right)=h\left(n T_{p}\right) \cdot W\left(n T_{p}\right)
$$

where $W\left(n T_{p}\right)$ is the Kaiser window 


$$
\begin{aligned}
W\left(k T_{p}\right)= & \frac{I_{0}\left(\alpha \sqrt{1-\left[\frac{(M-1-2 k) T_{p}}{(M-1) T_{p}}\right]^{2}}\right)}{I_{0}(\alpha)} \\
& \quad 0 \leq k \leq \frac{M-1}{2} \\
= & W\left((M+1-k) T_{p}\right) ; \quad \frac{M+1}{2} \leq k \leq M-1 \\
= & 0 ; \quad k<0 ; \quad k>M-1
\end{aligned}
$$

where

$$
\begin{aligned}
\alpha= & 0.1102(a-8.7) ; a>50 \\
\alpha= & 0.5842(a-21)^{0.4} \\
& +0.07886(a-21) ; \quad 21<a \leq 50
\end{aligned}
$$

and

$$
I_{0}(x)=1+\sum_{k=1}^{\infty}\left[\frac{(x / 2)^{k}}{k !}\right]^{2} .
$$

The parameter $a$ determines the shape of the Kaiser window as shown in Fig. 5.

Using a Kaiser window with $a=28$, the pass-band ripple is decreased to $1 \mathrm{~dB}$ peak to peak, conforming to the previously defined requirement. It also decreases the ripple in the stop-band which improves the attenuation in the stop-band. However, the transition between pass- and stopband becomes less steep, which is a disadvantage of the application of a window. The low-frequency results are presented in Fig. 6. From this figure, it can be seen that the chosen cutoff frequencies are pushed up to higher values by the windowing operation. The obtained stop-band attenuation of $22 \mathrm{~dB}$ needs further improvement, which involves methods that will also influence the cutoff frequency. Therefore, the stop-band attenuation will first be increased and then the influence on the cutoff frequency considered.

\section{Improvement of the Stop-Band Attenuation}

A simple method for improving the attenuation in the stopbands consists of correcting the impulse-response coefficients so that the stop-band amplification has its minimum, being zero, at the desired frequencies $f=0, f_{p}$, and $2 f_{p}$. This method can be explained by the spectrum shown in Fig. 4. All spectra mentioned in this text can be described as (17)

$$
H(f)=H^{\prime}(f) e^{-j 2 \pi f[(M-1) / 2] T_{p}} .
$$

In (17), $H^{\prime}(f)$ is real. Fig. 4 shows such a real part of a spectrum, where it can be seen that the stop-bands do not touch the $H^{\prime}(f)=0$ level but cross it so that the attenuation decreases.

The whole spectrum is now lifted by adding a certain constant factor $q$ so that $H^{\prime}(o)=H^{\prime}\left(f_{p}\right)=H^{\prime}\left(2 f_{p}\right)=0$ and the increased pass-band amplification is corrected by multiplying with $1 /(1+q)$ so that it again becomes one [see (18)].
In formula (19), the corrected spectrum $H_{\text {cor }}(f)$ is obtained from the existing spectrum, after windowing, $H_{w}(f)$

$$
\begin{aligned}
& H_{w}(f)=H_{w}^{\prime}(f) e^{-j 2 \pi f(M-1) / 2] T_{p}} \\
& H_{\text {cor }}(f)=\frac{1}{1+q}\left(H_{w}^{\prime}(f)+q\right) e^{-j 2 \pi f(M-1) / 2] T_{p}} .
\end{aligned}
$$

Thus, the existing impulse response coefficients $h_{w}\left(n T_{p}\right)$ are changed in the following way into a new impulse response $h_{\text {cor }}\left(n T_{p}\right)$ :

$$
\begin{aligned}
h_{\text {cor }}\left(n T_{p}\right)= & \frac{1}{1+q} h_{w}\left(n T_{p}\right) ; \\
& 0 \leq n \leq \frac{M-3}{2}, \quad \frac{M+1}{2} \leq n \leq M-1 \\
= & \frac{1}{1+q}\left(h_{w}\left(n T_{p}\right)+q\right) ; \quad n=\frac{M-1}{2} \\
= & 0 \quad \text { anywhere else }
\end{aligned}
$$

where

$$
q=-\sum_{n=0}^{M-1} h_{w}\left(n T_{p}\right)
$$

which means that the amplification for $f=0$ is exactly zero since

$$
\sum_{n=0}^{M-1} h_{\mathrm{cor}}\left(n T_{p}\right)=0 .
$$

Because of the spectrum periodicity, this is also true for $f=50 ; 100,150, \cdots, \mathrm{Hz}$.

The low-frequency part of the resulting spectrum is presented in Fig. 7.

\section{Limitations of the Cutoff Frequency}

There are some limitations on the value of the low cutoff frequency $f_{k}$ when considering the above-mentioned number of filter coefficients and filter method.

Choosing $f_{k}$ to be less than $0.7 \mathrm{~Hz}$ is not effective because then the frequency characteristics meet, as can be seen from Fig. 8. A steeper edge can only be achieved by using a greater impulse response length, which implies more filter coefficients and therefore more superfluous computational operations.

Fig. 8 also shows the low-frequency behavior of the filter for values of $f_{k}>1.5 \mathrm{~Hz}$. When $f_{k}>1.7 \mathrm{~Hz}$, the ripple in the stop-band increases strongly, minimizing the minimum attenuation in the stop-band. Increasing parameter $a$ of the Kaiser window can minimize this ripple, but more filter coefficients will be necessary in order to retain the transition edge steepness. The above-mentioned considerations and previous investigations [2] led to the following specifications:

$$
\begin{aligned}
& M=51 \\
& T_{p}=1 / 50 \mathrm{~s} .
\end{aligned}
$$




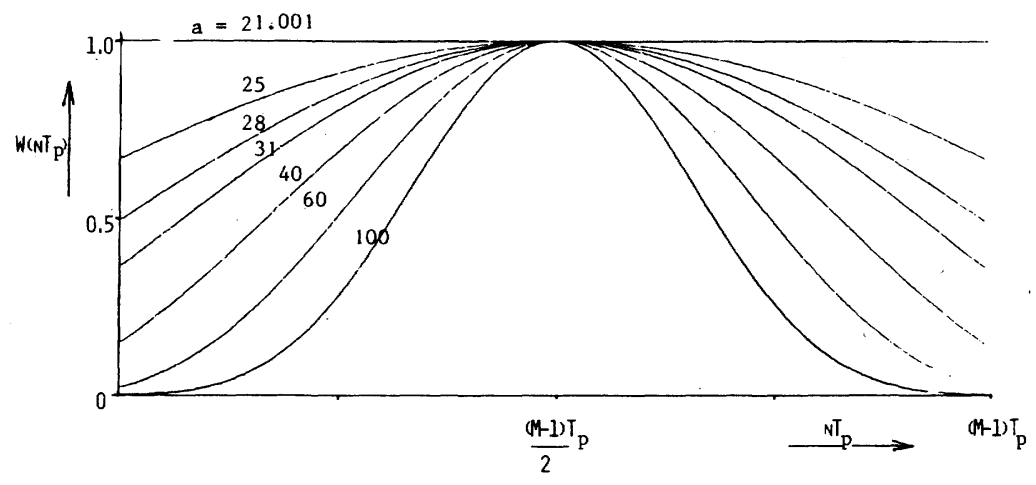

Fig. 5. The (continuous) shape of the Kaiser window and its dependency on parameter $a$.

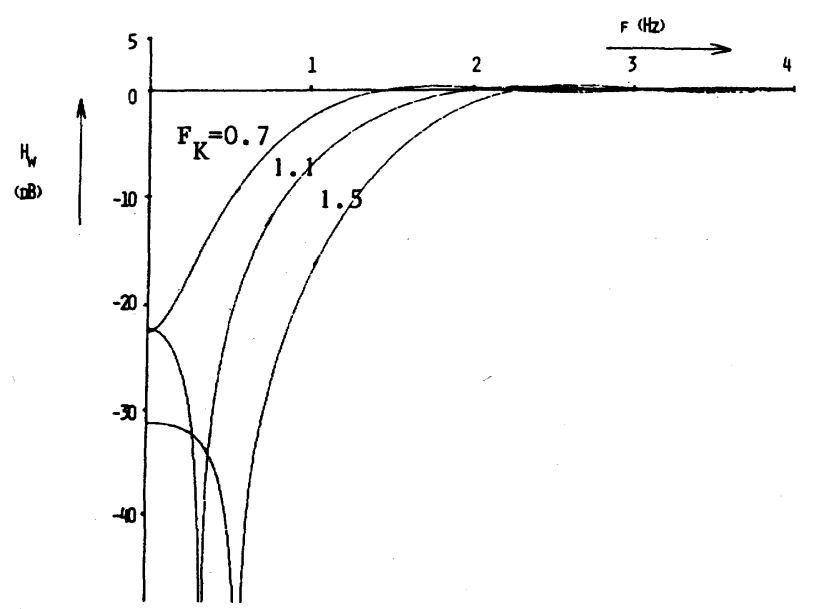

Fig. 6. Low-frequency behavior of the filter after truncation of the impulse response to $1.0 \mathrm{~s}$ and multiplication by a Kaiser window with $a=28$. Values of $f_{k}$ (see Fig. 1) were chosen as: $f_{k}=0.7,1.1$, and $1.5 \mathrm{~Hz}$.

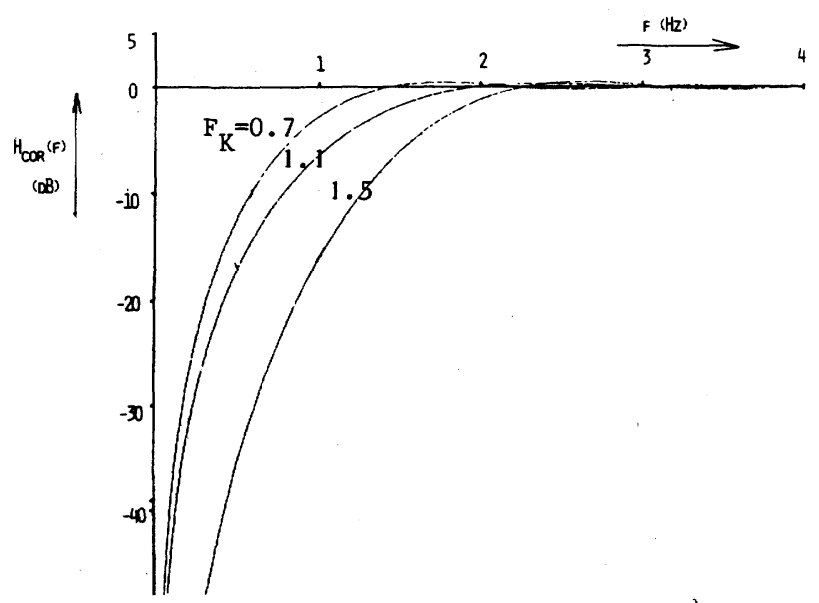

Fig. 7. Low-frequency behavior of the filter as presented in Fig. 6 but now after improvement of the attenuation in the stop-bands $f=0,50,100$, $\cdots, \mathrm{Hz}$.

These values correspond to an impulse response length of $1.0 \mathrm{~s}$. The cutoff frequency $f_{k}$ may vary from 0.7 to $1.5 \mathrm{~Hz}$ without invalidating the specifications.

\section{Application of the Filtering to Exercise ECG's}

Some results of the filtering of the ECG are presented in Figs. 9-11. These figures show the ECG before the fil-

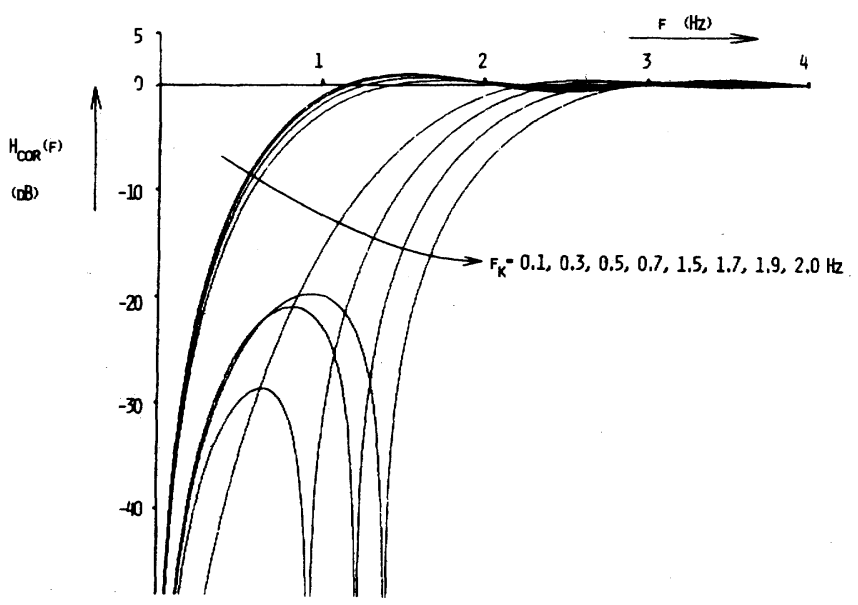

Fig. 8. Low-frequency behavior of filters corrected with a Kaiser window $(a=28)$ and improved stop-band attenuation for various values of the cutoff frequency $f_{k}$.

tering operation, the ECG after filtering but corrected for the filter delay, and the difference between the input and output signals, being the part of the original ECG signal that is removed. The plots clearly show that the base-line wander has been removed. It can be seen from the "thicker" parts of the difference signal that $50 \mathrm{~Hz}$ components have also been removed.

A problem is how much to filter, i.e., what belongs to the "true" ECG which may not be removed. As a criterion for not disturbing the ECG, it was agreed that the difference signal (input-output) may not contain components appearing with the same period as the ECG beats [2]. Fig. 12 shows a filtered ECG where the cutoff frequency is too high and the difference signal contains components correlating with ECG beat periods.

\section{ConClusions}

The introduced method for removal of base-line wander and mains frequency components from the ECG consisting of NRFIR filtering, with a reduced number of filter coefficients, gives good results.

The number of filter coefficients is reduced by a factor of 5 compared to conventional NRFIR filtering without loss of spectral properties. The number of multiplications is again halved by using the impulse-response symmetry. 


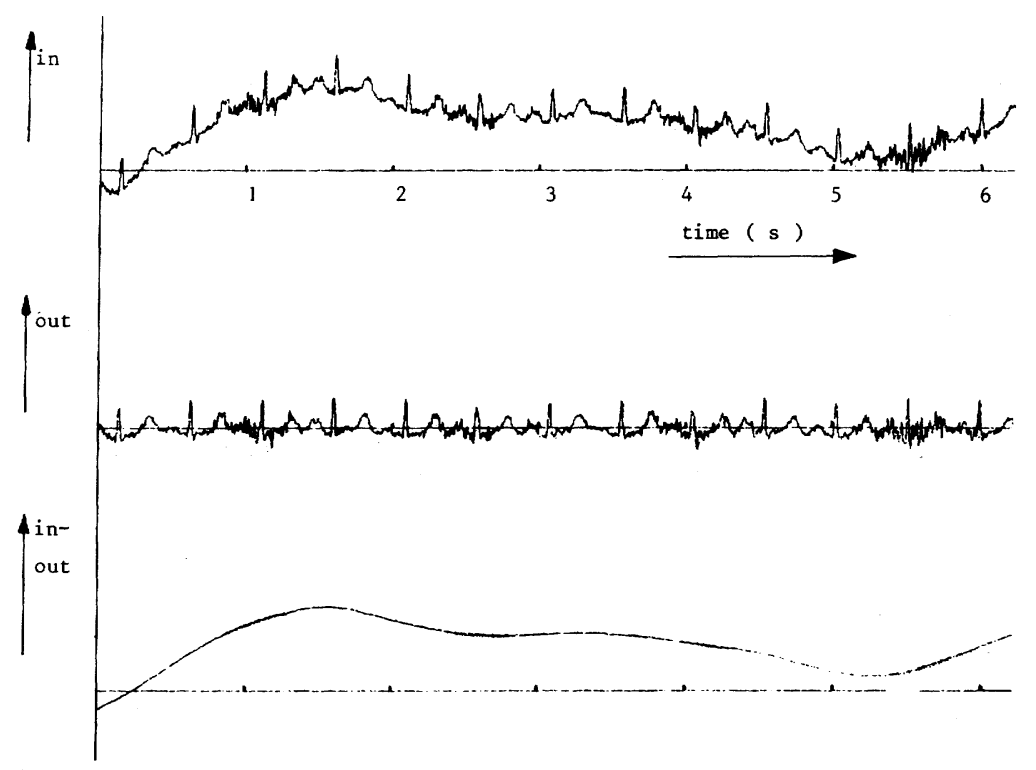

Fig. 9. An example of the influence of digital filtering of an ECG registration distorted by base-line wander and myopotentials. The input signal (in), the output signal (out) and the removed part of the input signal (inout) are shown. The signals are corrected for the delay that is introduced in the output signal by the filter.

Filter specifications:

inpulse response length $=1.0 \mathrm{~s}, 51$ coefficients, $f_{k}=0.7 \mathrm{~Hz}$,

$H(f)=0$ for $f=0,50,100 \mathrm{~Hz}$,

Kaiser window $(a=28)$ used, and periodic spectrum.

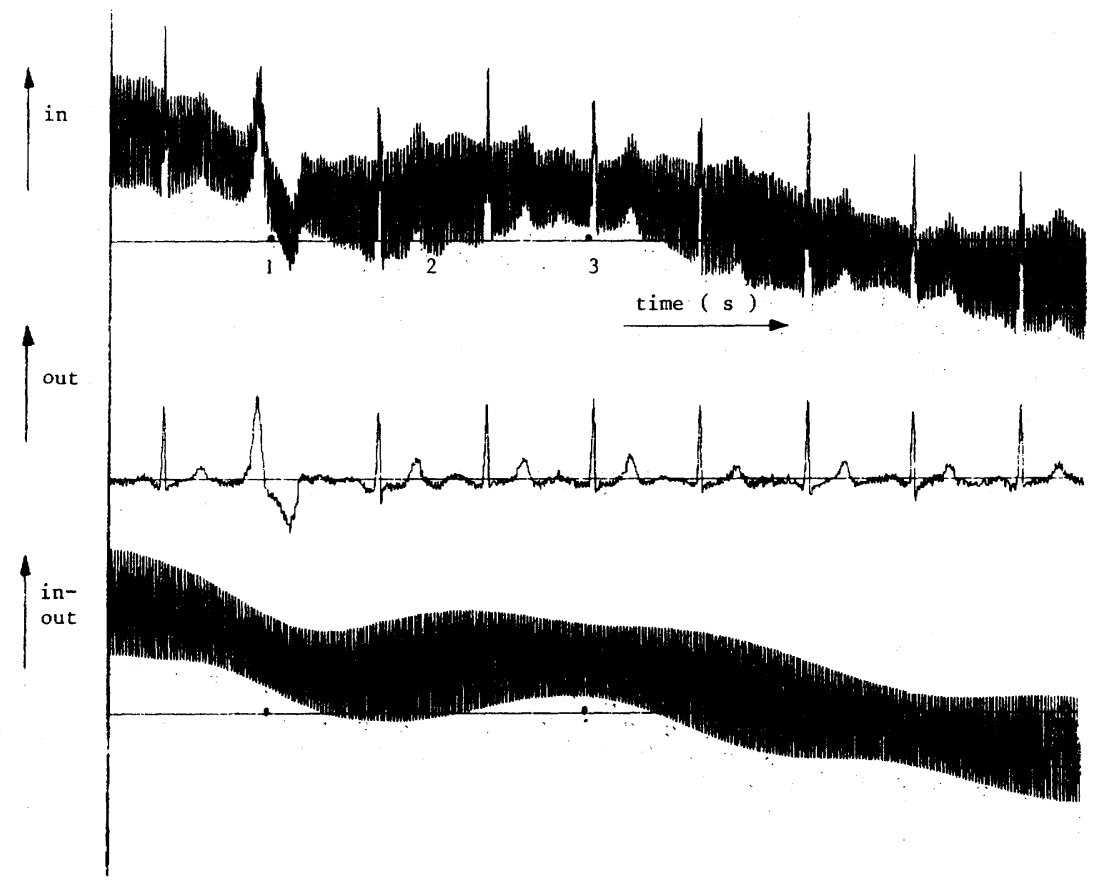

Fig. 10. An example of filtering of an ECG containing ectopic beats. The input signal is distorted by artificial $50 \mathrm{~Hz}$ power-line interference.

Filter specifications:

impulse response length $=1.0 \mathrm{~s}, 51$ coefficients,

$f_{k}=0.7 \mathrm{~Hz}$

$H(f)=0$ for $f=0,50,100 \mathrm{~Hz}$,

Kaiser window ( $a=28$ ) used, and periodic spectrum. 


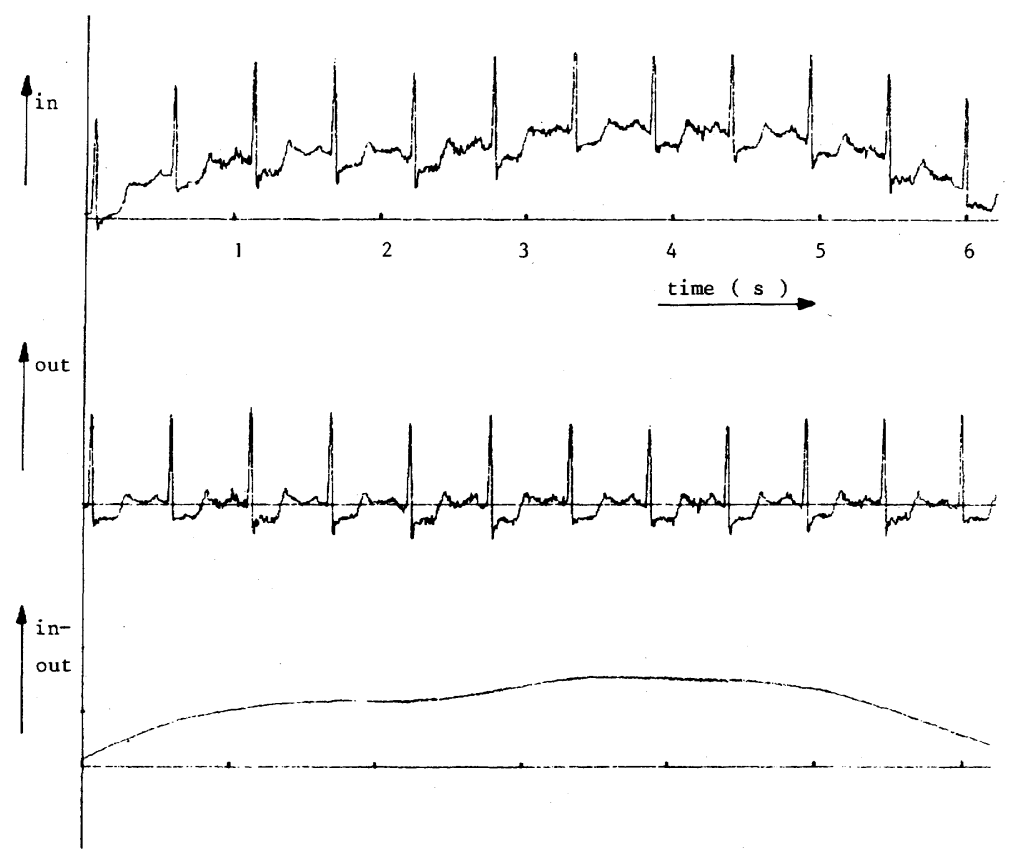

Fig. 11. An example of filtering of an ECG containing normal beats with $S T$-segment depression.

Filter specifications:

impulse response length $=1.0 \mathrm{~s}, 51$ coefficients

$f_{k}=0.7 \mathrm{~Hz}$,

$H(f)=0$ for $f=0,50,100 \mathrm{~Hz}$,

Kaiser window $(a=28)$ used, and periodic spectrum.

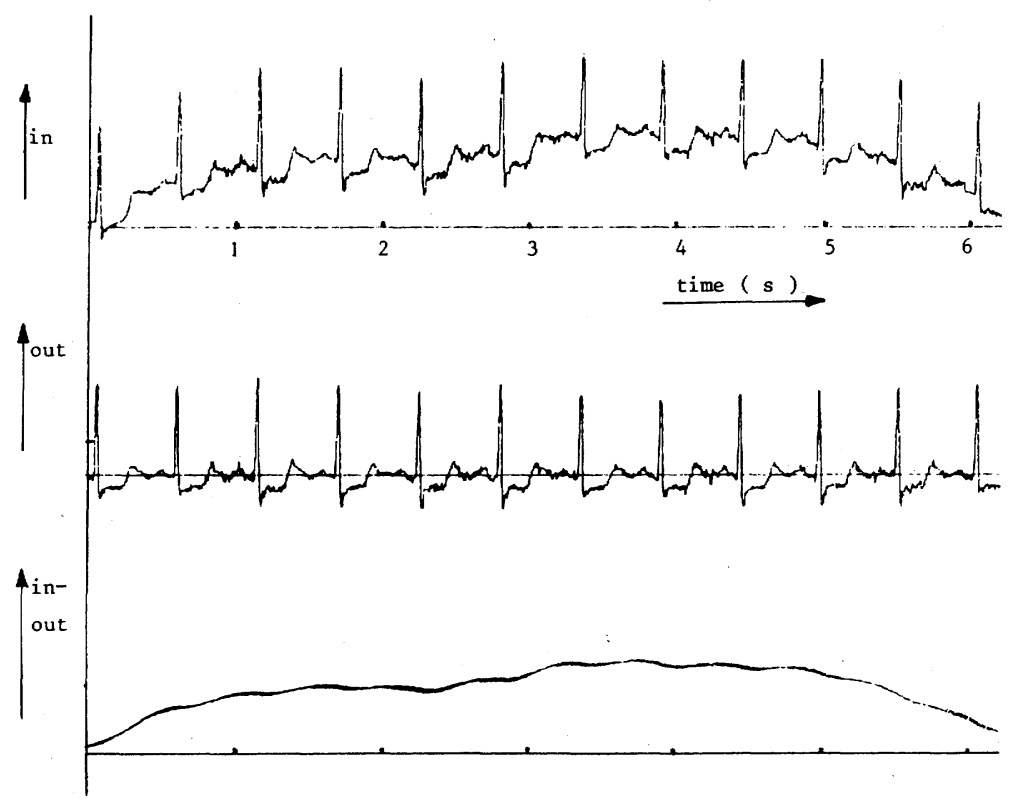

Fig. 12. An example of ECG filtering where the low cutoff frequency is too high $\left(f_{k}=1.5 \mathrm{~Hz}\right)$. In the difference signal, a periodic phenomenon can be observed correlated with the heartbeat occurrences. Due to the increased width of $3 \mathrm{~Hz}$ of the stop-band at $50 \mathrm{~Hz}$ and $100 \mathrm{~Hz}$, these frequency components become more dominant in the difference signal.

Filter specifications:

impulse response length $=1.0 \mathrm{~s}, 51$ coefficients,

$f_{k}=1.5 \mathrm{~Hz}$,

$H(f)=0$ for $f=0,50,100 \mathrm{~Hz}$,

Kaiser wndow $(a=28)$ used, and periodic spectrum. 
The total number of multiplications needed to process an ECG, that is, sampled at a rate of $250 \mathrm{~Hz}$, with a filter having an impulse length of $1.0 \mathrm{~s}$, is reduced to 26 per sample. This means that the ECG filtering operation for three channels simultaneously can be performed in real time by general purpose microcomputers such as the Motorola 68000 or the DEC LSI 11/23, without the necessity of extra hardware.

\section{REFERENCES}

[1] C. R. Meijer and H. N. Keiser, "Electrocardiogram noise estimation and removal using cubic splines and state-space computation techniques," Comput. Biomed. Res., vol. 10, pp. 459-470, 1977.

[2] J. A. van Alsté, W. van Eck, and O. E. Herrmann, "Methods for electrocardiography in patients unable to perform leg exercise: Rowing ergometry, robust averaging and linear phase filtering," in Computers in Cardiology. Florence, Italy: IEEE Computer Society, 1981, pp. 465468.
[3] J. P. Marques de Sa, "Digital FIR filtering for removal of ECG baseline wander," J. Clin. Eng., vol. 8, pp: 235-240, 1982.

[4] A. V. Oppenheim and R. W. Schafer, Digital Signal Processing. Englewood Cliffs, NJ: Prentice-Hall, 1975.

[5] American Heart Associations Committee on Electrocardiography, "Recommendations for standardization of leads and of specifications for instruments in ECG/VCG," Circulation, vol. 52, pp. 11-25, 1975.

J. A. Van Alsté (S'75-S'83-M'83-M'84), photograph and biography not available at the time of publication.

T. S. Schilder, photograph and biography not available at the time of publication. 